\title{
Un même paradigme pour la réponse immunitaire au virus de l'hépatite C et au VIH ?
}

\section{Yves Lévy}

Unité d'immunologie clinique, $\mathrm{CHU}$ HenriMondor, 51, avenue du Maréchal-de-Lattrede-Tassigny, 94010 Créteil Cedex, France et Inserm U. 474, Maternité Port-Royal, 123, boulevard de Port Royal, 75014 'Paris, France.

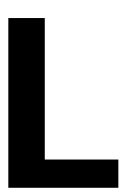

'infection par le virus de I'hépatite C (VHC) est un problème majeur de Santé Publique puisque près de 170 millions d'individus sont infectés dans le monde.

Si I'hépatite aiguë liée au VHC est le plus souvent asymptomatique, dans la majorité des cas, le virus persiste après infection et peut être responsable d'une hépatite chronique, de cirrhose, ou de cancer du foie. Les mécanismes responsables de la persistance virale ou des lésions hépatiques sont inconnus.

Les données récentes concernant la dynamique de la replication virale et la réponse immunitaire lors de I'infection aiguë ou chronique par le VHC sont proches des caractéristiques de l'infection par le VIH. En effet, chez les sujets infectés par ces virus de nombreux variants viraux sont produits immédiatement après la phase aiguë de l'infection. L'hépatite aiguë due au VHC, comme la primoinfection par le VIH, peut être asymptomatique. Ceci explique l'absence de données concernant la cinétique et la spécificité de la réponse immunitaire lors de l'infection aiguë au VHC. Les manifestations pathologiques liées à ces deux virus apparaissent plusieurs années après
I'infection. Dans l'infection chronique par le $\mathrm{VHC}$, et le $\mathrm{VIH}$, une réponse immunitaire impliquant des lymphocytes $\mathrm{T} \mathrm{CD}^{+}$cytotoxiques spécifiques du virus a pu être mise en évidence [1-3]. Cependant, celle-ci reste insuffisante pour éliminer le virus. La réponse immunitaire antivirale peut être absente du sang et être localisée soit dans le foie pour le $\mathrm{VHC}$, soit dans les ganglions ou le tube digestif pour le VIH, rendant difficile le suivi de cette réponse immunitaire. Cependant, l'obtention de ces données est indispensable pour tenter d'établir une corrélation entre les effecteurs de la réponse immunitaire et le devenir de l'infection virale, l'élimination ou non du virus et le lien avec les lésions pathologiques hépatiques.

L'analyse des séquences des gènes codant pour l'enveloppe du VHC lors de l'infection aiguë, chez 12 patients, a permis d'établir une corrélation entre la dynamique de la replication virale, I'apparition de variants du VHC (quasi-espèce), et l'évolution clinique [4]. Les patients infectés par un VHC ayant une forte capacité à produire des variants viraux lors de l'infection aiguë sont incapables d'éliminer le virus et ont tendance à développer une infection chronique au VHC, contrairement aux patients chez lesquels le virus évolue peu. Ces résultats suggèrent, comme cela est proposé pour l'infection par le VIH, que la diversité des populations virales lors de l'infection aiguë est dépendante de la qualité, et de la nature, de la réponse immunitaire de I'hôte [5]. En effet, une réponse immunitaire optimale lors de l'infection aiguë diminuerait le risque d'apparition de variants du VHC facilitant ainsi son élimination. Cependant, le caractère asymptomatique de la majorité des infections aiguës par le VHC a longtemps gêné l'analyse de la réponse immunitaire des patients infectés. Récemment, le groupe de B. Walker a pu étudier 3 patients au cours de l'infection aiguë [6]. Chez ces sujets, une réponse $\mathrm{T} C \mathrm{CD} 8^{+}$cytotoxique, spécifique de plusieurs épitopes du VHC [2-8], a pu être détectée dans le sang par I'utilisation de tétramères de molécule HLA de classe I HLA-A2 liés aux épitopes du VHC. Ceci signifie qu'une réponse immunitaire restreinte par d'autres molécules HLA de classe I de I'hôte reste possible, ce qui augmenterait considérablement la polyspécificité des cellules cytotoxiques. Ces lymphocytes $T$ $\mathrm{CD}^{+}{ }^{+}$comme les lymphocytes $\mathrm{T}$ 
$\mathrm{CD}^{+}$spécifiques également mis en évidence chez ces patients, synthétisent de I'Interféron $\gamma$ après activation par les épitopes viraux. L'analyse longitudinale montre la persistance des réponses immunitaires $\mathrm{T} \mathrm{CD8}^{+}$et $\mathrm{CD}^{+}$chez ces sujets après disparition de la virémie du VHC et guérison de l'hépatite.

Cette observation rappelle les données obtenues par le même groupe lors de la primo-infection par le $\mathrm{VIH}$, au cours de laquelle une forte réponse immunitaire spécifique $\mathrm{T}$ $\mathrm{CD}^{+}$et $\mathrm{CD}^{+}$est détectable chez les patients. Cependant, à long terme, ces effecteurs T CD4 ${ }^{+}$spécifiques disparaissent et ne seront plus détectés à nouveau chez les patients, malgré I'augmentation du taux des lymphocytes $\mathrm{T} \mathrm{CD}^{+}$mémoires lié à introduction des thérapeutiques antirétrovirales. Or la persistance à long terme des réponses $\mathrm{T} \mathrm{CD}^{+}$spécifiques est indispensable au maintien des réponses effectrices $\mathrm{CD}^{+}$capables de contrôler la replication virale. Les raisons pour lesquelles ces cellules disparaissent ne sont pas connues: absence "d'antigènes » en raison d'une parfaite inhibition de la replication virale par les traitements antirétroviraux? anergie des cellules $\mathrm{T}$ $\mathrm{CD}^{+}{ }^{+}$spécifiques? disparition des cellules T $\mathrm{CD}^{+}{ }^{+}$spécifiques du sang, où elles sont recherchées pour des raisons évidentes de faisabilité par la majorité des équipes? délétion clonale? L'étude de la réponse immunitaire d'une cohorte de femmes infectées par le VHC après perfusion d'immunoglobulines antirhésus dans le cadre de la prévention de la maladie hémolytique du nouveau-né, a permis d'étudier l'évolution de la réponse immunitaire, humorale et cellulaire à long terme [7]. Parmi 77 patientes, 43 ont pu éliminer le virus après une infection aiguë tandis que 34 sont chroniquement infectées 18 à 20 ans après avoir été exposées au VHC. Une grande fraction des patientes guéries (42\%) n'a plus d'anticorps anti-VHC détectables, contrairement aux patientes chroniquement infectées. La mise en évidence $d$ 'une réponse humorale sur des prélèvements congelés 10 ans auparavant confirme bien qu'il s'agit d'une disparition des réponses humorales chez les patientes guéries. En revanche, une réponse immunitaire spécifique $\mathrm{T} \mathrm{CD}^{+}$et $\mathrm{CD}^{+}$reste détectable chez la majorité des patientes guéries. Ceci contraste clairement avec l'observation d'une disparition de ces réponses chez les patientes ayant une réplication chronique du VHC. Ces résultats sont concordants avec ceux rapportés par le groupe de B. Walker [6] montrant la disparition des cellules $\mathrm{T} \mathrm{CD}^{+}$ spécifiques du VHC chez les patients avec hépatite chronique alors que la fréquence de ces cellules peut être élevée (jusqu'à 4\% des lymphocytes $\mathrm{T} \mathrm{CD}^{+}$) chez les patients ayant éliminé le virus.

Quels sont les mécanismes immunologiques expliquant le maintien d'une réponse immunitaire cellulaire chez les patients ayant éliminé le VHC? De même, quels sont les mécanismes de la perte de la réponse immunitaire chez les patients ayant une replication chronique du virus? Malgré I'absence d'ARN du VHC détectable dans les cellules mononucléées du sang des patients guéris, l'existence $d^{\prime}$ une replication virale à bas bruit dans le foie et responsable du maintien d'une réponse immunitaire ne peut pas être éliminée. Cependant les lymphocytes $\mathrm{T} \mathrm{CD8}^{+}$spécifiques du VHC semblent appartenir à un compartiment de cellules mémoires non activées (absence d'expression des molécules HLA de classe II), ce qui pour les auteurs élimine I'hypothèse $\mathrm{d}^{\prime}$ une production récente in vivo de ces cellules et privilégie plutôt des cellules mémoires à longue vie [6] Plusieurs études ont montré chez des modèles animaux la possibilité de maintenir à long terme, en l'absence d'antigène spécifique, des cellules mémoires capables d'activité effectrices lors d'une stimulation spécifique [8]. Enfin, la disparition des cellules $\mathrm{T}^{\mathrm{CD}} 8^{+}$spécifiques chez les patients ayant une replication chronique du VHC soulève I'hypothèse d'un échappement des variants viraux au système immunitaire, mécanisme décrit lors de l'infection chronique par le $\mathrm{VIH}$. Un rôle «immunomodulateur» à long terme du VHC interférant soit avec l'activation des cellules spécifiques, soit avec la présentation de I'antigène peut également être évoqué. Ce fait n'est pas sans rappeler la capacité du gène nef du VIH à diminuer l'expression des molécules HLA de classe I par les cellules infectées, gênant ainsi la présentation des épitopes viraux au système immunitaire. Ainsi, en totale analogie avec ce que I'on pense aujourd'hui pour l'infection par le $\mathrm{VIH}$, il est possible que l'infection aiguë par le VHC stimule une réponse lymphocytaire $\mathrm{T} \mathrm{CD}^{+}$ spécifique permettant la production de cellules $\mathrm{T} \mathrm{CD}^{+}$antivirales indispensables à l'élimination du virus chez les patients qui guérissent de I'hépatite. Si cette réponse immunitaire est insuffisante, ou si le virus persiste, ces effecteurs peuvent progressivement disparaître. Ceci suggère qu'une stratégie d'immunothérapie spécifique, combinée au traitement actuellement proposé chez les patients chroniquement infectés par le VHC (association d'Interféron $\alpha$ et d'un médicament antiviral comme la RIBAVIRINE), pourrait être utile

\section{RÉFÉRENCES}

1. Koziel MJ, Dudley D, Afdahal N, et al. HLA class I-restricted cytotoxic T lymphocytes specific for hepatitis $C$ virus. Identification of multiple epitopes and characterization of patterns of cytokine release. J Clin Invest 1995; 96: 2311-21.

2. Rehermann B, Chang K, McHutchinson J, et al. Differential CTL responsiveness to hepatitis B and $C$ viruses in chronically infected patients. J Virol 1996; 70: 7092-102.

3. Rehermann B, Chang K, McHutchinson J, Kokka R, Houghton M, Chisari F. Quantitative analysis of the peripheral blood CTL response in patients with chronique HCV infection. J Clin Invest 1996; 98: 1432-40.

4. Farci P, Shimoda A, Coiana A, et al. The outcome of acute hepatitis $C$ predicted by the evolution of the viral quasipecies. Science 2000 ; 288: 339-44.

5. Pantaleo G, Demarest J, Schacker T, et al. The qualitative nature of the primary immune response to the primary immune response to HIV infection is a prognosticator of disease progression independent of the initial level of plasma viraemia. Proc Natl Acad SCi USA 1997; 94 : 254-9.

6. Lechner F, Wong DKH, Dunbar PR, et al. Analysis of successful immune responses in persons infected with hepatitis C virus. J Exp Med 2000; 191: 1499-512.

7. Takaki A, Wiese M, Maertens G, et al. Cellular immune responses persist and humoral responses decrease two decades after recovery from a single-source outbreak of hepatitis C. Nat Med 2000; 6: 578-82.

8. Murali-Krishna K, Lau LL, Sambhara $\mathrm{S}$, Lemonnier F, Altman J, Ahmed R. Persistence of memory Ć $8^{+} \mathrm{T}$ cells in MHC class I-deficient mice. Science 1999; 286: 1377-81.

\section{TIRÉS À PART}

Y. Lévy. 